\title{
Religión y política en palabras de Ramon Llull*
}

\author{
Enzo Solari** \\ Pontificia Universidad Católica de Valparaíso \\ Valparaíso, Chile
}

Para citar este artículo: Solari, Enzo. «Religión y política en palabras de Ramon Llull».

Franciscanum 176, Vol. 63 (2021): 1-18.

\section{Resumen}

Es capital, en toda la monumental obra de Llull, la forma lingüística de plantear la disputatio interreligiosa y la alegorización política. Todo esto se aprecia en el Llibre del gentil e dels tres savis y en el Llibre de les bèsties, con distintos pero retóricamente eficaces recursos. Esta breve comunicación está dedicada a mostrar la importancia de estas obras para cumplir mediante variadísimos estilos lingüísticos con el sempiterno propósito luliano de argumentar la verdad cristiana y convertir a los que aún no la han aceptado.

\section{Palabras clave}

Llull, lenguaje, religiones, política, razón.

\section{Religion and politics in the words of Ramon Llull}

\begin{abstract}
The question of language is decisive through the impressive Llull's work, and it is so also when he offers an interreligious disputatio and a political allegorization. All this accent is noted in the Llibre del gentil e dels tres savis and in the Llibre de les bèsties, with rhetorically different but effective resources. This paper pretends to show the importance of these works to achieve, by means of diverse linguistic styles, the everlasting purpose of Llull: to argue the Christian truth and to convert the people that not yet accept that.
\end{abstract}

\section{Keywords}

Llull, language, religions, politics, reason.

\footnotetext{
${ }^{*}$ Este texto es parte de una investigación sobre la tolerancia medieval financiada por el Proyecto Fondecyt $\mathrm{N}^{\circ}$ 11090205. Una primera versión de este texto fue leída en el $\mathrm{XV}^{\circ}$ Congreso Latinoamericano de Filosofía Medieval, celebrado en abril de 2015 y organizado por las Facultades de Filosofía y Teología de la Pontificia Universidad Católica de Santiago de Chile.

** Licenciado en Ciencias Jurídicas, Magíster en Filosofía de la Pontificia Universidad Católica de Valparaíso y Doctor en Filosofía por la Hochschule für Philosophie/Philosophische Fakultät SJ de München, Alemania. Profesor de la Facultad de Derecho de la Pontificia Universidad Católica de Valparaíso. ORCID: https://orcid.org/0000-0002-5713-761X. Contacto: enzo.solari@pucv.cl.
} 


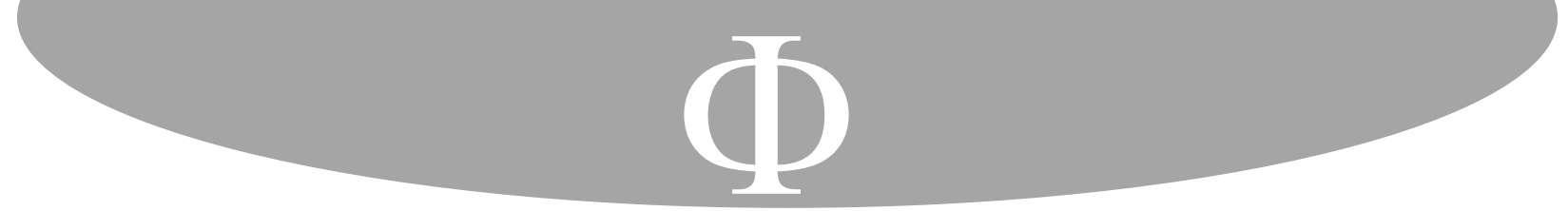

todos tengamos una sola fe y una ley por la que podamos dar a Dios nuestro Señor, creador de todos los bienes, la gloria y alabanza debidas ${ }^{13}$.

La clave para llegar a un acuerdo está en recurrir no a autoridades, sino a «razones demostrativas y necesarias» ${ }^{14}$. El gentil está de acuerdo: requiere demostraciones para creer en Dios, la creación y la resurrección: «quien pueda demostrarme a mí la resurrección con razones vivas y concluyentes, podría arrancar de mi alma el dolor que sufre (...) Porque cada día me voy aproximando a la muerte y pienso que no habrá nada después de la muerte» ${ }^{15}$. Uno de los sabios, entonces, dice que es preciso investigar mediante algunas flores para probar al gentil la existencia de Dios, sus virtudes y la resurrección, y a través de los cinco árboles demostrarle lo que es necesario y obligatorio que sepa (demonstremus gentili hoc ad quod scire necessario est obligatus) ${ }^{16}$.

En el libro primero, dice el narrador, «se demuestra con razones necesarias que Dios existe y que en él existen las flores del primer árbol y la resurrección» ${ }^{17}$. En plena demostración mediante las flores del primer árbol, el gentil -turbado por la ignorancia"comenzó a esclarecerse con la luz de la verdad» y a alegrarse ${ }^{18}$. Al cabo de ese libro el gentil «recuerda e intelige» las razones esgrimidas, los árboles y sus flores, y la luz (coruscatio) del esplendor divino, que ilumina su intelecto, da a su corazón el dulce apetito por el que desea llegar a la vía salutífera. El gentil entonces, pasando de la ignorantia a la notitia, se libera de tormentos y tristezas y se goza y alegra y bendice a los tres sabios ${ }^{19}$. Pero también piensa en su tierra, padre y madre y en su error e infidelidad, y entonces pide a los sabios compasión por los que están en el error, no tienen noticia de Dios ni le dan gracias por sus beneficios. Ellos debieran ir a honrar a Dios entre quienes no lo honran, predicando allí la verdad y adoctrinándolo a él mismo para que pueda dirigir en el camino de la salvación a las gentes que se hallan en el camino del fuego ${ }^{20}$. En ese momento cada uno de los sabios le responde que se convierta a su fe y a su ley, con lo que se produce una contradicción entre los tres. El gentil pregunta si hay una ley mejor o si las tres son verdaderas, pero cada sabio alaba su propia fe y reprocha la de los demás. Entonces el gentil vuelve a entristecerse con dolores y tristezas aún mayores que las originales ${ }^{21}$. Pide a los sabios que discutieran ante él con razones para poder discernir quién de ellos está en la vía salutífera. Los sabios aceptan discutir libremente para determinar al cabo la verdad y el error ${ }^{22}$. La clave, dice un sabio, está en seguir el mismo método con el que los tres han llegado a demostrar la existencia de Dios, sus virtudes y la futura resurrección: tratar de probar la concordancia de los artículos de la fe de cada uno «con las flores y condiciones de aquellos árboles». Así se determinará cuál es la

\footnotetext{
${ }^{13}$ Ramon Llull, Libro del gentil y los tres sabios, 24-27.

${ }^{14}$ Ramon Llull, Libro del gentil y los tres sabios, 26-27.

${ }^{15}$ Ramon Llull, Libro del gentil y los tres sabios, 30-31.

16 Véase Ramon Llull, Libro del gentil y los tres sabios, 32-33.

${ }^{17}$ Ramon Llull, Libro del gentil y los tres sabios, 10-11.

${ }^{18}$ Véase Ramon Llull, Libro del gentil y los tres sabios, 54-55.

${ }^{19}$ Véase Ramon Llull, Libro del gentil y los tres sabios, 115 y 117.

${ }^{20}$ Véase Ramon Llull, Libro del gentil y los tres sabios, 118-119.

${ }^{21}$ Véase Ramon Llull, Libro del gentil y los tres sabios, 120-121.

22 Véase Ramon Llull, Libro del gentil y los tres sabios, 122-123.
} 


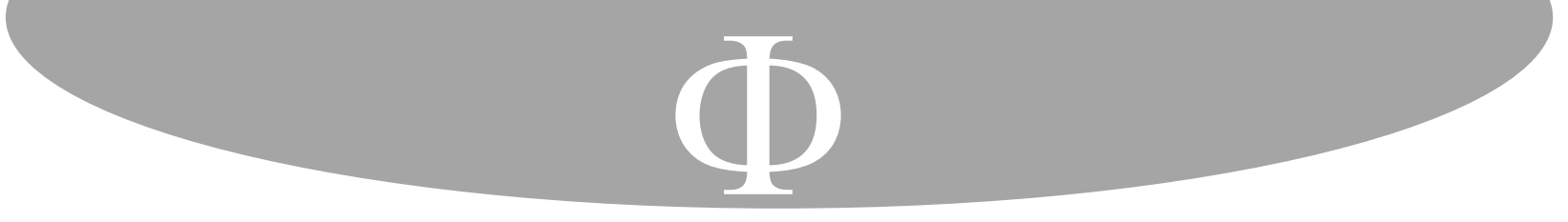

religiones monoteístas, [aunque por otra parte...] ingenuamente se confía en la posibilidad de reducirlas a la unidad por el Arte, la buena fe y la inteligencia» ${ }^{54}$. Y es que no hay que perder de vista que «comprender el mecanismo interno del ars es presuposición para la lectura de la mayoría de las obras de Llull», y que por lo mismo el Libro del gentil permite ver también la profunda peculiaridad del Arte luliano frente a las corrientes filosóficoteológicas de su época, sobre todo porque ars es ese terminus technicus que designa aquella ciencia general que contiene a la teología y a la filosofía, las cuales por ende ya no son ciencias independientes una de otra ${ }^{55}$. Expresiva y temáticamente, por lo demás, es sabido que el Libro del gentil es tributario del Kuzari de Halevi y del Dialogus de Abelardo, entre otros, y también -a través de la alambicada tradición del lulismo-que influye en el Cusano y en tantos más. Allende el Libro del gentil, el uso luliano del lenguaje es soberano y sumamente diverso, tanto así que se ha dicho que Llull es el don Quijote catalán ${ }^{56}$.

Pero conviene ser más específicos. En el Libro del gentil las razones argumentadas intentan ser persuasivas y eficaces. Esta dimensión retórica también fue tematizada por Llull. La Retòrica nova (Rhetorica nova, de 1301-1303) aborda el uso persuasivo del lenguaje, y podría ser leída como la autocomprensión de la variable lingüística y de la constante apologética de la entera obra luliana. Este autocomentario se vuelve sobre su producción anterior, la de las obras fechables entre 1271 y 1301 -entre las cuales está el Libro del gentil y también el Fèlix o Llibre de meravelles. Según la Retòrica nova, de cuatro partes consta la retórica: ordo, pulchritudo, scientia y charitas $^{57}$. En este sentido, «la nueva retórica que propone Ramon Llull podría ser el resultado de un largo examen de conciencia sobre esta actividad, la de 'encontrar' libros complementaria del Arte, que no se ha de apartar de un orden que produce belleza si comunica la ciencia con caridad» ${ }^{58}$. Es esta una investigación de las principales características necesarias para producir toda clase de parlamentos o discursos (en latín, sermones). Cuando Llull trata de la belleza en la parte 2, apartado 4, de la Nueva retórica, ofrece 24 ejemplos, dentro de los cuales recuerda fábulas del Fèlix hasta hoy sumamente eficaces comunicativamente, en lo que constituye -repito- una suerte de autocomentario luliano para el cual la belleza según la retórica consiste en la eficacia comunicativa $^{59}$.

\footnotetext{
${ }^{54}$ Lola Badia, Teoría y practica de la literatura en Ramon Llull (Barcelona: Quaderns Crema, 1991), 12 y 2528.

${ }^{55}$ Fernando Domínguez y Jorge Uscatescu, «Ramon Llull», 1074-1075 y 1077.

${ }^{56}$ La idea viene de Menéndez y Pelayo, y recientemente ha sido repetida: «la vida de Ramon Llull es tan inverosímil que resulta tentador considerarlo el don Quijote catalán, un personaje literario en lugar de una figura histórica. Él es, de hecho, el Cervantes de Cataluña, su Goethe, su Dante, su Chaucer, el creador que inaugura un lenguaje literario y funda así una tradición. Fue verdaderamente quijotesco, aunque su Dulcinea fue la conversión voluntaria de los judíos y los musulmanes, una desquiciada empresa en la que Llull no tuvo a ningún Sancho Panza que le hiciera entrar en razón (...) El visionario catalán no fue un personaje prudente, a la manera en que tampoco lo sería Giordano Bruno, solo que Llull fue tan católico como Bruno hermético»: Harold Bloom, Ramon Llull and catalan tradition (Barcelona: Institut Ramon Llull, 2006), 171-205, aquí 173.

${ }^{57}$ Mientras las cinco partes convencionales de la retórica eran inventio, dispositio, elocutio, memoria y actio: cf. Lola Badia, «Nova retòrica i pràctica d'escriptura en Ramon Llull», Quaderns d'Italià 18 (2013): 79-91, aquí 86. Sigo a Badia a propósito de la retórica luliana.

${ }^{58}$ Lola Badia, «Nova retòrica i pràctica d'escriptura en Ramon Llull», Retòrica, 91.

${ }^{59}$ Véase Lola Badia, «Nova retòrica i pràctica d'escriptura en Ramon Llull», 86 y 80.
} 


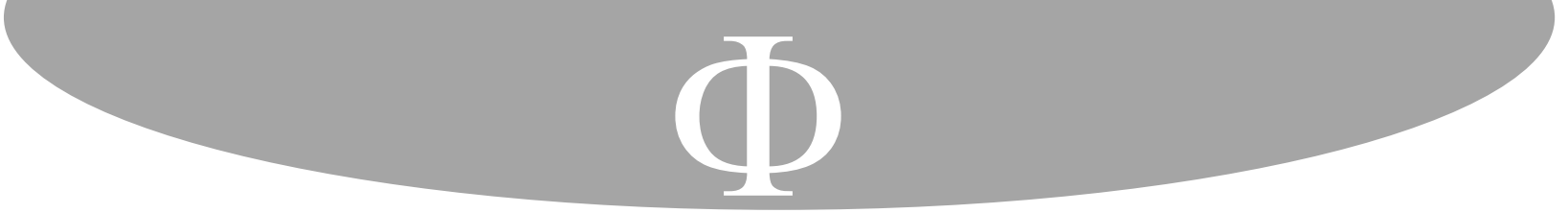

Precisamente en el Libro de las maravillas aparece el Llibre de les bèsties, que es tanto una fábula animalística como también un espejo de príncipes ${ }^{60}$. En vez del utopismo del Libro del gentil o de la perspectiva evaluativa del Ars brevis (recuérdese su definición de la política: «el arte con el que los ciudadanos [burgenses] procuran el bien público de la ciudad [publicam utilitatem civitatum] ${ }^{61}$ ), este excepcional texto de Llull parece mostrar una antropología pesimista («la peor bestia y la más falsa que haya en el mundo es el hombre» ${ }^{62}$ ) y por lo mismo una aguda consciencia de las estrategias, mentiras y aun violencias de la Realpolitik. Este libro, se ha dicho, bebe de varios modelos y parece adelantar otros tantos. Por ej., en la elección del rey, relatada en el primer capítulo, se aprecia la influencia del hebreo Libro de los jueces, 9,1-15, y de la carta 90 de Séneca a Lucilio. Por otro lado, cierto maquiavelismo ha sido varias veces destacado, pues este libro enseña (siguiendo otro espejo de príncipes de origen indio: Calila y Dimna) que la astucia o maestria consigue lo que no alcanza la fuerza. La zorra ( $N a$ Renard) cuenta al león -el rey-la historia de un cuervo y una gran serpiente mucho más fuerte que aquel, pero que termina siendo derrotada (y perdiendo la vida) por la industria o maestria del cuervo: «tengo yo tal arte e ingenio, que si llegara a ocurrir que no pudiese vencer por fuerza de armas (...), me valdría de arte e ingenio (jo he tanta d'art i de maestria, que si s'esdevenia que no pogués vèncer per força d'armes (...), aidar-men hauria per art e per maestria)» ${ }^{63}$. A la vez, este libro no enseña pero sí muestra satíricamente cómo es que un rey puede comportarse desaforada, despóticamente, cómo la traición, la ruptura de la fidelidad ocurren en política una y otra vez (véase sobre todo el capítulo 42 del Libro de los animales, cuando la zorra aconseja al rey que no mantenga la palabra empeñada ${ }^{64}$ ).

Esto, claro, es semejante a lo que posteriormente recomendará Maquiavelo en el capítulo 18 de Il Principe:

\footnotetext{
${ }^{60}$ Véase Josep Ruiz, «De guineus, lleons, llops i pastors: domini i govern en el pensament polític de Llull», Quaderns d'Italià 18 (2013): 157-178, aquí 162. En gral. y en breve sobre los espejos de príncipe, véase Joaquín Abellán, Conceptos políticos fundamentales (Madrid: Alianza, 2012), 80-85.

${ }^{61}$ Ramon Llull, «Arte breve», 316-317. Del utopismo del Libro del gentil y de Llull en gral. mucho se ha escrito. Por todos, véase Joaquín Xirau, «Vida y obra de Ramón Llull. Filosofía y mística (1946)», Obras completas Vol. 2 (Barcelona: Anthropos, 1999), 215-349, especialmente 317-349, Miguel Cruz Hernández, El pensamiento de Ramon Llull (Madrid: Castalia, 1977), 205-252, José Luis Abellán, «IX. La filosofía catalana: Ramon Llull», Historia crítica del pensamiento español Vol. 1: Metodología e introducción histórica (Madrid: Espasa-Calpe, 1979) 290-293. Para los Carreras i Artau, Llull es el gran utopista de la época, sobre todo por el Libre de Blanquerna y (¡también!) por el Llibre de meravelles: véase Tomàs Carreras i Artau y Joaquim Carreras i Artau, Història de la filosofía española. Filosofia cristiana del segle XIII al XV Vol. 1: El escolasticismo popular. Ramon Lull (Raimundo Lulio) (Barcelona/Girona: Institut d'estudis catalans/Diputació de Girona, 2001), 630-634.

${ }^{62}$ Ramon Llull, Félix o Libro de maravillas, trad. J. Ribera (Madrid: BAC, 2016), 185.

${ }^{63}$ Ramon Llull, Félix o Libro de maravillas, 180.

${ }^{64}$ Véase Julia Butiñá, «Sobre el escandaloso 'Llibre de les bésties’ de Ramón Llull y su audiencia», Espacio, Tiempo y Forma, Serie III, Historia Medieval 17 (2004): 79-94, aquí 86-91. Véase Ramón Llull, Obra, 172180.
} 


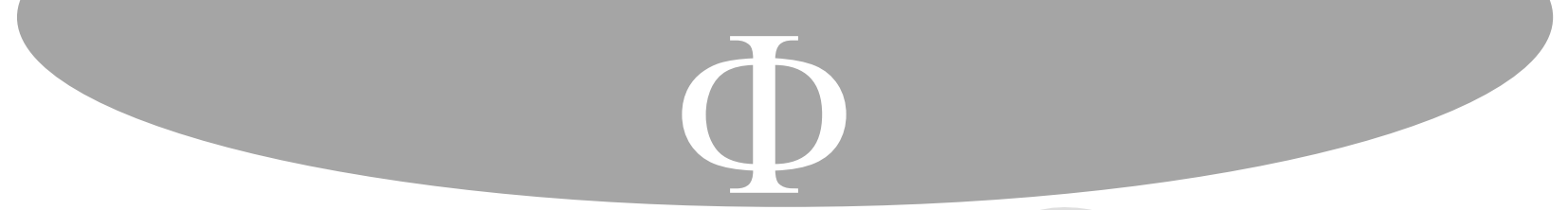

Por observaciones como las anteriores es que se ha dicho que la obra (siempre teológica) de Llull adopta esa peculiar definición de hombre como animal homificans: precisamente para enfatizar su libertad, su pluralidad y diferencia, y la consiguiente necesidad humana de paz y unidad ${ }^{69}$. La cual, por lo demás, es un rasgo decisivo del pensamiento de Llull, cuya «fe en el diálogo y en el entendimiento pacífico» se habría seguido afirmando inquebrantablemente ${ }^{70}$.

\section{Conclusión}

Pues bien, en los plurales recursos lingüísticos lulianos se entremezclan política y religión con tonos a veces anticipatorios de posteriores vías modernas. No obstante lo cual, toda esta diversidad lingüística, religiosa y política, y todos los aires de familia que comparte Llull con renacentistas y humanistas, no pueden ocultar cuán medieval fue el de Mallorca y cuán unitariamente enraizados en el Arte están sus textos. Más bien habría que decir que Llull tuvo una absorbente preocupación, la de difundir el evangelio cristiano y por ello convertir a los infieles o, como dice en la Vita coetanea (obra de su madurez fechable en 1311), «dar su vida por honor de Jesucristo, y escribir (...) libros (...) y hacer construir y edificar diversos monasterios ${ }^{71}$. Para cumplir con tal misión usó de todos los medios a su alcance, medios que fueron muchos y muy distintos unos de otros, pero en todos los cuales se reflejaba esa misma intención. En la misma Vita coetanea se trasluce la unidad de su empeño mediante la diversidad de sus manifestaciones. En el Libro del gentil Llull escenifica una tolerante y deseable disputa religiosa, aprecia la imparcialidad (= regla de oro ${ }^{72}$, confía en las razones

trayecto, descubre al hombre en su papel de pacificator»: Julián Barenstein, Homo pacificator. El hombre y la paz en Ramon Llull y Giovanni Pico della Mirandola, 119-120.

69 «Como Dios solo es Dios trinitariamente, haciéndose Dios, el hombre sólo es hombre haciéndose hombre (homificans), en ese irse haciendo hombre y cuanto más hombre más perfecto para alcanzar y comprender el ser humano su específico modo de ser. Esa comprensión, ese saberse hombre, es el comienzo y fin de toda actividad humana (...) El pensamiento luliano libre de autoridades y de magisterio escolar, libre también de la tutela clerical, es una ciencia que, aunque Llull no la llevó a sus últimos extremos filosóficos, abría un horizonte insospechado. Aquel horizonte que los humanistas supieron traducir en un nuevo concepto de libertad de estudio e investigación. Libertad no significa ya que el hombre ha recibido de la naturaleza un ser cierto y terminado que va llevando de alquiler a través de su vida, sino algo que se alcanza, se supera y se realiza en un continuo hacer, hacerse y superarse. De ahí aquel homo est animal homificans que en traducción muy libre podríamos transcribir así: el hombre ha recibido un alma (racional), es decir, es un ser animado que (conscientemente) vive (en un proceso) homificante (porque es) homificable (es decir perfeccionable) para continuamente homificarse a sí mismo y a los demás seres corporales y para humanizarse más a sí mismo y humanizar el mundo que le rodea (...) Está claro que Ramon Llull es un ejemplo a seguir en la aceptación radical del otro. Nada existiría para Llull sin la pluralidad, sin la diversidad de colores, sonidos, lenguas, religiones, razas... La aceptación de esa condición del ser es constitutiva de todo su pensamiento y es por ello que su pensamiento se puede ofrecer como punto de partida y ejemplar diseño de un diálogo productivo dentro de la diversidad de culturas»: Fernando Domínguez, «Dios, el mundo y el hombre según Ramon Llull», Revista de lenguas y literaturas catalana, gallega y vasca 20 (2015): 245-258, aquí 255-256.

${ }^{70}$ Jordi Gayà, «Introducción», en Ramon Llull, Félix o Libro de maravillas, Introducción, XXXV.

${ }^{71}$ Ramón Llull, Obra, 5 (Vita N ${ }^{\circ} 7$ ).

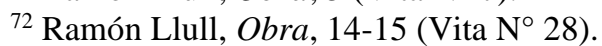


